\section{In-stent Restenosis in an Anomalous Left Main Coronary Artery Arising from the Right Sinus of Valsalva After a Stenting Lesion with Acute Angle}

\author{
Wail Alkashkari 1, 2, 3 , Alaa Meer ${ }^{4}$, Attafah Omeish ${ }^{4}$, Mohammed Althobaiti ${ }^{5}$, Abdulhalim J. Kinsara ${ }^{6}$ \\ 1. Cardiology, King Faisal Cardiac Center, King Abdulaziz Medical City, Jeddah, SAU 2. Cardiology, King Saud Bin \\ Abdulaziz University for Health Science, Jeddah, SAU 3. Medical Research, King Abdullah International Medical \\ Research Center, Jeddah, SAU 4. Cardiology, King Faisal Cardiac Center, King Abdulaziz Medical City, Ministry of \\ National Guard Health Affairs, Jeddah, SAU 5. Radiology, King Abdulaziz Medical City, Ministry of National Guard \\ Health Affairs, King Abdullah International Medical Research Center, King Saud Bin Abdulaziz University for Health \\ Science, Jeddah, SAU 6. Cardiology, Ministry of National Guard Health Affairs, King Abdullah International Medical \\ Research Center, King Saud Bin Abdulaziz University for Health Sciences, King Abdullah International Research Center, \\ Jeddah, SAU
}

Corresponding author:Wail Alkashkari, wakash73@hotmail.com
Received 02/13/2020 Review began 03/04/2020 Review ended 03/05/2020 Published 03/08/2020

\section{() Copyright 2020}

Alkashkari et al. This is an open access article distributed under the terms of the Creative Commons Attribution License CC-BY 4.0., which permits unrestricted use, distribution, and reproduction in any medium, provided the original author and source are credited.

\begin{abstract}
We describe a 38-year-old male who underwent percutaneous coronary intervention (PCI) using a thirdgeneration drug-eluting stent (DES) with a thin stent for an anomalous left main coronary artery (LMCA) originating from the right coronary sinus with a retro-aortic course. Six months later, in-stent restenosis (ISR) occurred due to stent implantation in angled lesions with significant hinge motion. An intravascular ultrasound (IVUS) revealed significant neointimal hyperplasia. The vessel wall of an angled coronary artery lesion is exposed to mechanical stress from the deployed stent. It has been reported before in the major coronary arteries but not in an anomalous LMCA.

Categories: Cardiology

Keywords: restenosis, angioplasty, balloon, coronary, drug-eluting stent

\section{Introduction}

An angled coronary lesion, which is often accompanied by hinge motion, is reportedly a risk factor for instent restenosis (ISR) with all generations of coronary stents [1]. Several mechanisms are behind the higher incidence of ISR in angled lesions [2]. Initially, it was described after bare-metal stent (BMS) implantation. Drug-eluting stents (DES) have dramatically reduced the rate of ISR as compared with BMS. However, ISR in angled lesions remains a problem, especially with old-generation DES having a closed-cell, stainless-steel design, which has low conformability and flexibility. Recently, third-generation DES, which have thin stent struts and an open-cell design, lead to better conformability and flexibility at the expense of weak radial strength and the risk of stent fracture, especially at an angled lesion. Several studies have reported the relationship between angled lesions with hinge motion and ISR after DES implantation [3]. We describe a case of ISR due to an acute angle lesion with hinge motion after third-generation DES implantation for the first time in an anomalous LMCA.
\end{abstract}

\section{Case Presentation}

A 38-year-old male patient with a 15-pack per year smoking history presented to another hospital with acute anterolateral ST-elevation myocardial infarction. He was thrombolyzed with t-PA with the complete resolution of his electrocardiography (ECG) changes. Echocardiogram revealed mild left ventricular (LV) dysfunction with a left ventricular ejection fraction (LVEF) of $40 \%-45 \%$ and anterior/lateral wall hypokinesis. He was then transferred to our center where he was taken to the catheterization laboratory and a diagnostic coronary angiogram was done after obtaining informed consent. The angiogram revealed a normal right coronary artery and an anomalous origin of the left main coronary artery from the right coronary cusp (Figure 1). The anomalous left main showed a mid-shaft hazy and eccentric lesion (Figure 2). Normal left anterior descending and normal left circumflex were present. The patient was discussed in a heart team meeting and it was decided to proceed with a coronary CT angiogram to define the course of the anomalous LMCA, which was retro-aortic. The patient's informed decision was to opt for percutaneous coronary intervention (PCI). A percutaneous intervention was carried out using a right Judkins 4 guiding catheter and a balance middle weight (BMW) wire that passed to the distal left anterior descending artery (LAD) and a direct 4.0x22 mm resolute stent deployed (Figure 3). A second 4.0x8 mm resolute stent was deployed proximally to cover an area of plaque shift. This was followed by post-stent balloon dilatation using a 4.5X15 mm non-complaint balloon at high pressure. A final intravascular ultrasound (IVUS) was done, which revealed good stent apposition (Figure 4). The patient discharged home within 48 hours [4]. 


\section{Cureus}

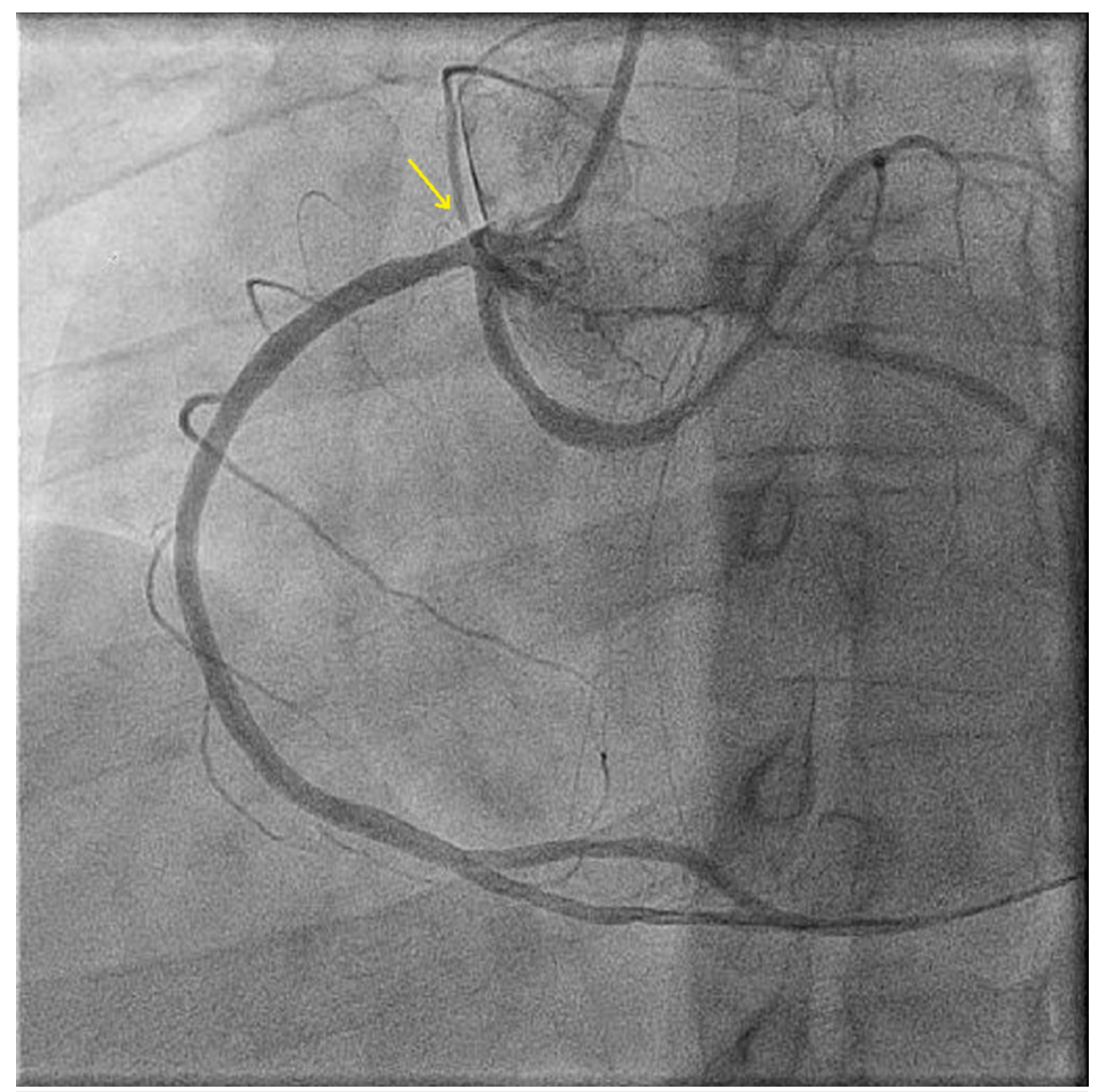

FIGURE 1: Coronary angiography showing right and left coronary arteries originating from the right coronary sinus of Valsalva 


\section{Cureus}

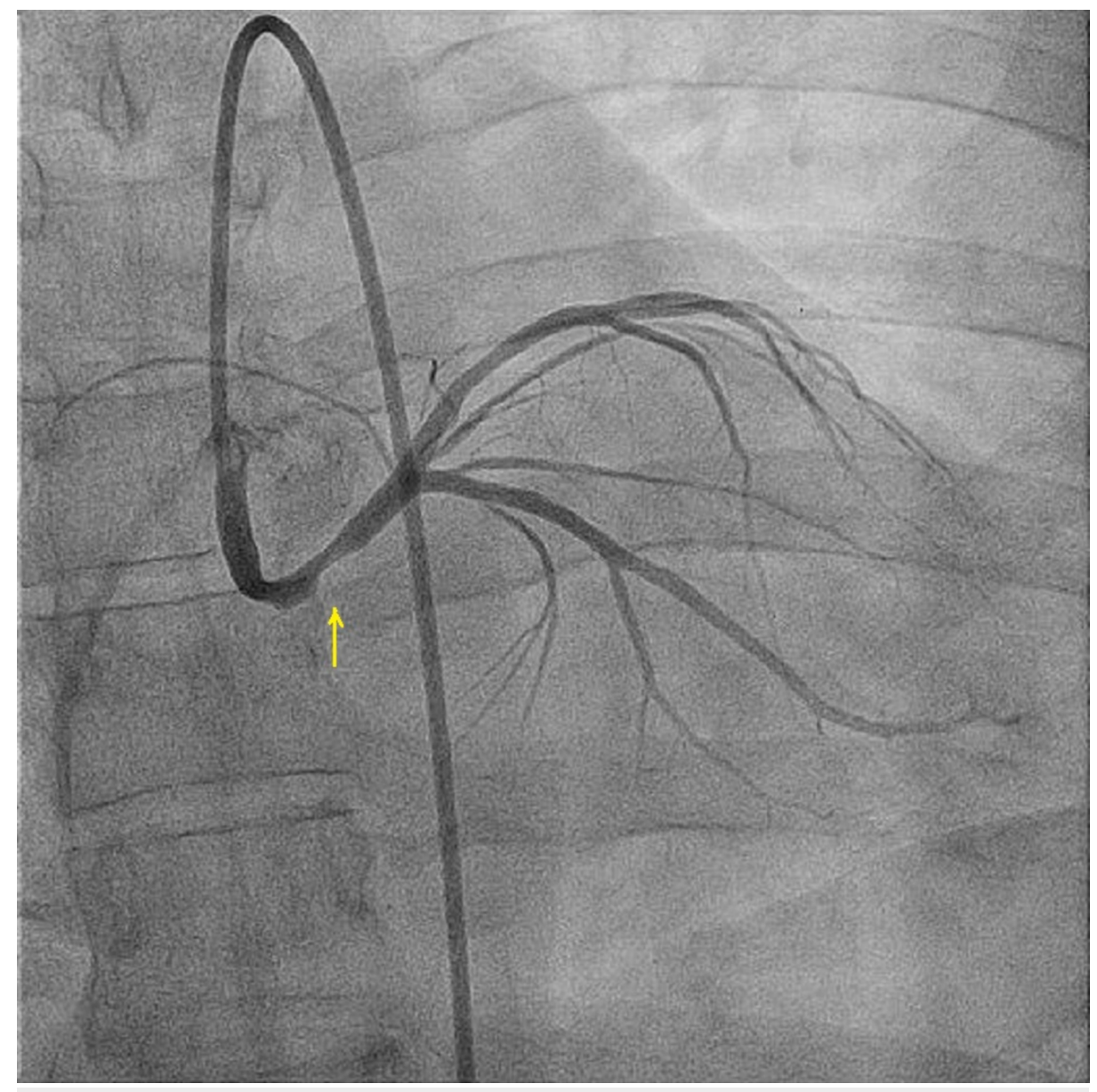

FIGURE 2: Coronary angiography showing right and left coronary arteries originating from the right coronary sinus of Valsalva 


\section{Cureus}

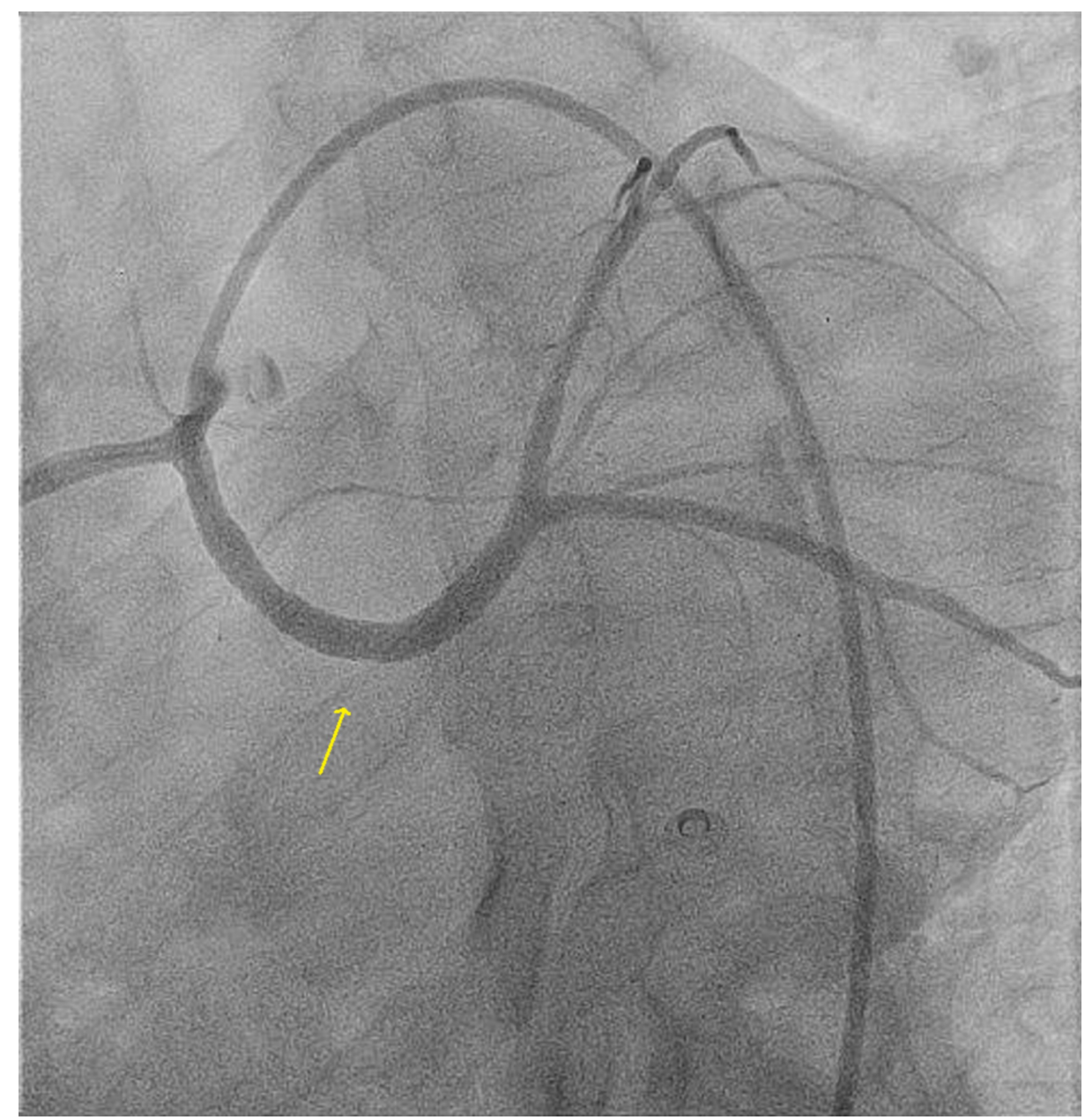

FIGURE 3: Post stenting of the anomalous left main 


\section{Cureus}

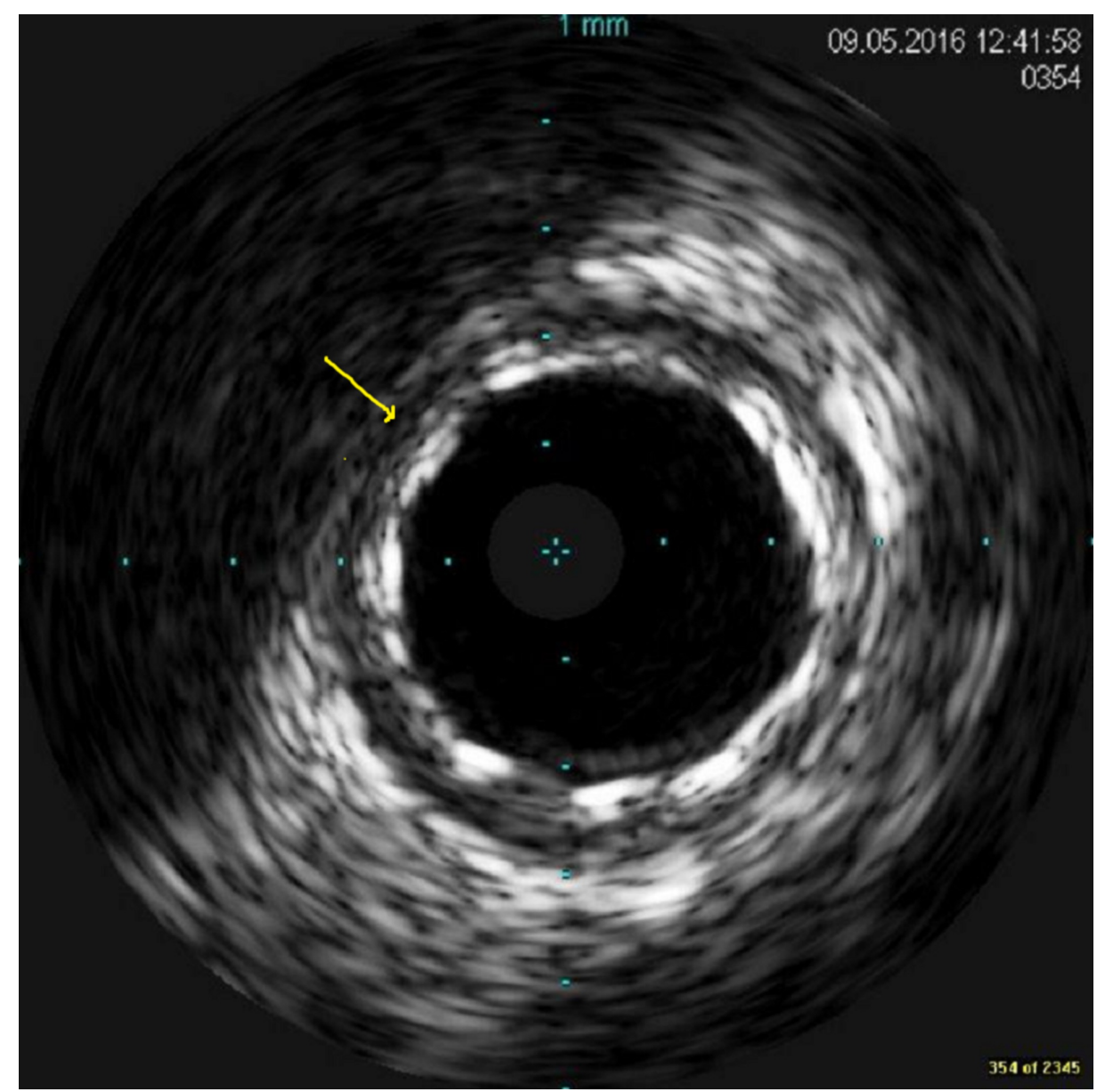

FIGURE 4: The final intravascular ultrasound image of the stented segment with excellent apposition

Six months later, he was admitted again due to the recurrence of chest pain with a normal ECG and mild troponin rise. The echocardiogram revealed an LVEF of $50 \%$. Of note, the patient quit smoking since discharge. The coronary angiogram revealed significant proliferative type three ISR (10 mm extending beyond the stent margins) (Figure 5). IVUS was done and revealed intimal hyperplasia with no evidence of an obvious stent fracture or malapposition. The patient was offered surgery but he refused. In view of the diffuse pattern of intimal hyperplasia and no stent/native artery size mismatch, we proceeded with the placement of the $4 \times 15 \mathrm{~mm}$ Xience stent (Abbott Laboratories, Lake Bluff, Illinois), followed by $4.5 \times 12 \mathrm{~mm}$ non-complaint balloon dilation at high pressure (Figure 6). The final IVUS revealed an excellent result. 


\section{Cureus}

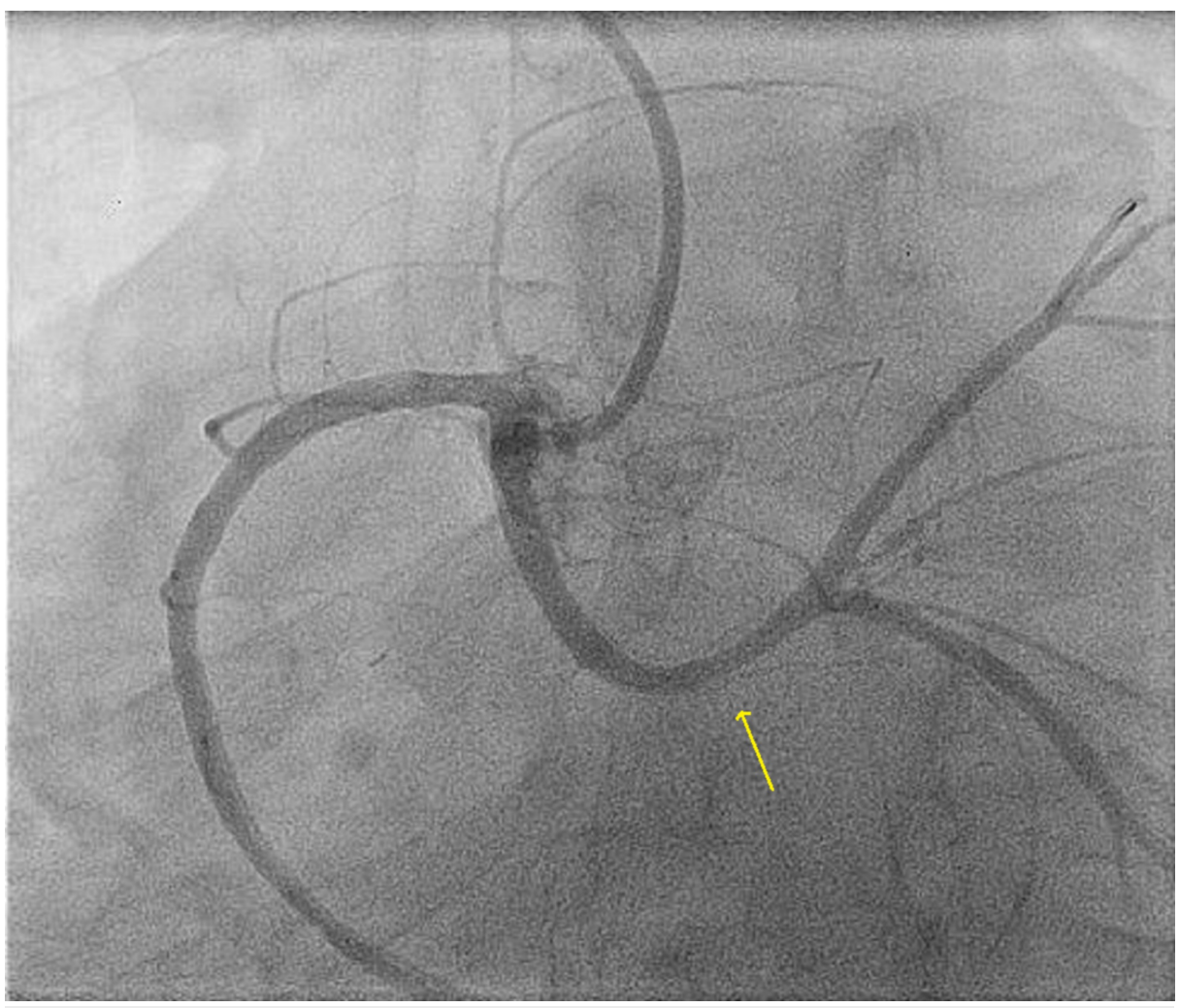

FIGURE 5: Type three left main in-stent restenosis

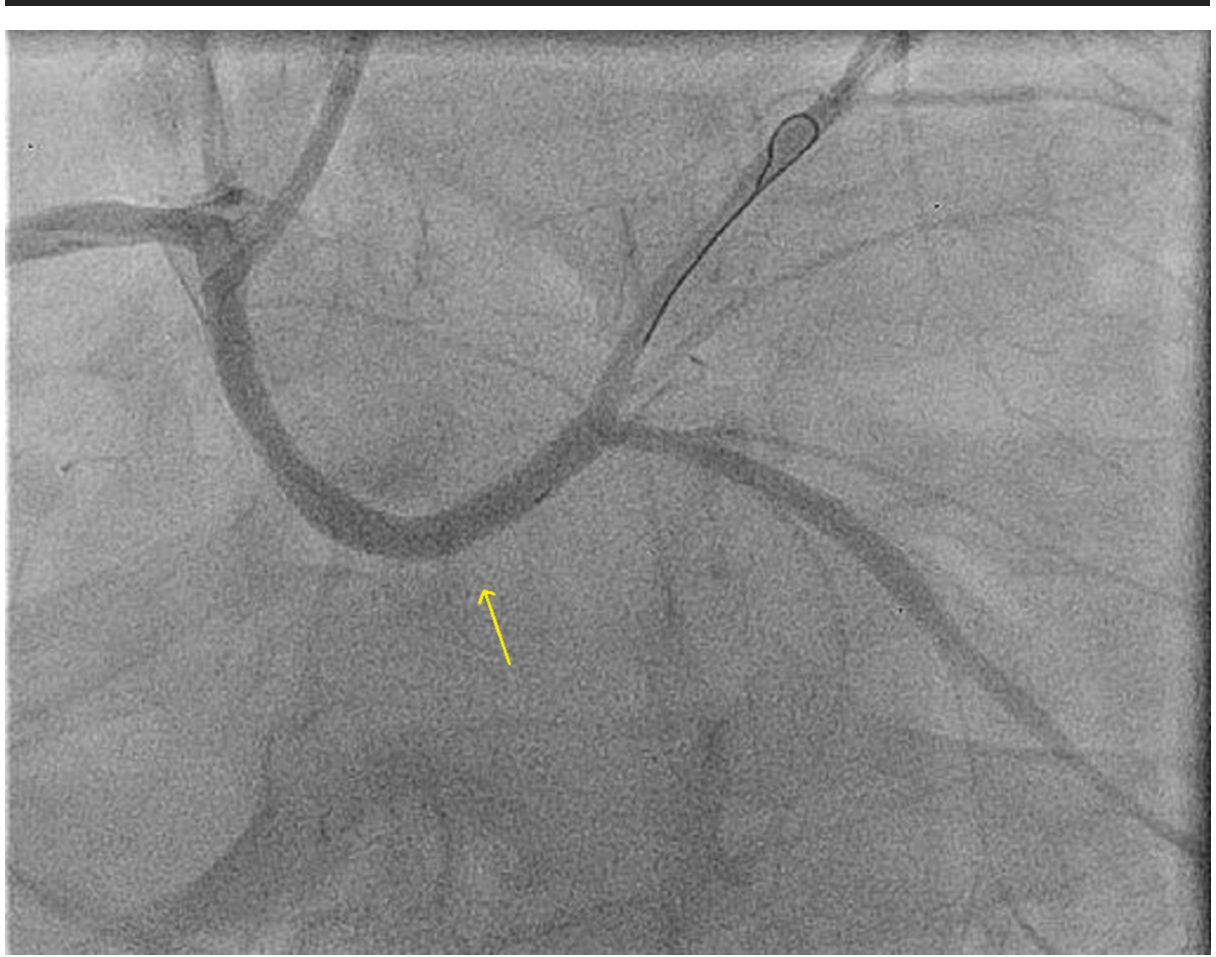

FIGURE 6: Post in-stent restenosis stenting

\section{Discussion}

The clinical incidence of ISR after BMS implantation is approximately 20\%-35\%. The use of DES has led to a further decrease in the occurrence of ISR to $5 \%-10 \%$ [5]. ISR after coronary angioplasty is currently one of the main limitations of this method, leading to the recurrence of exertional angina pectoris or acute coronary syndromes. Several risk factors contributed to the development of ISR [6]. These include patients, 


\section{Cureus}

lesions, and procedural factors (Table 1). Angled lesions had a higher incidence rate of ISA over non-angled lesions and were an independent predictor for ISA in several reports. There are several hypotheses behind ISR in angled lesions [6]. First is the mechanical stress (shearing force) from the stent strut to the vessel wall of angled lesions that provokes neointimal hyperplasia. Second, some struts in the inner curvature at the edge would not be well attached to the vessel wall in angled lesions even if the stent was well expanded with recommended balloon pressure. Third, stent recoil was observed in some stent designs. A recent report indicated that stent recoil after the first-generation DES occurred in $0 \%-2 \%$. Some speculate that stent recoil is prone to occur in hinge motion lesions because excessive vessel movement causes stent compression at the hinge point during the cardiac cycle, and stent recoil causes a decrease in minimal stent area. Fourth, a stent fracture had been reported as the reason for ISR in angled lesions in several studies. Furthermore, small stent fractures, such as a single strut fracture, which is difficult to detect with fluoroscopy or IVUS, might occur at the angled lesions. In one study, they found that the angle of $\geqslant 16^{\circ}$ is the optimal cut-off value for predicting ISR [2]. 


\section{Cureus}

\section{Risk Factors}

\section{Patient Factors}

Age

Female sex

Genetic factors

Diabetes mellitus

Renal insufficiency

Acute coronary syndrome

Lesion Factors

Long lesions

Small vessels

Complex B2/C lesions

Chronic closures

Ostial lesions

Bifurcation lesions

Lesions in venous bypass

Recurrent restenosis

Multivessel coronary artery disease

Angled lesions

Procedure Factors

Type of stent

Number of stents and total length

Stent overlap

Stent underexpansion

Stent fractures

Post-PCI MLD

\section{TABLE 1: In-stent restenosis risk factors}

PCI: percutaneous coronary intervention; MLD: minimal lumen diameter

The clinical significance of the high incidence of vessel wall injuries and ISA in angled lesions is still unclear. A recent clinical study of second-generation DES reported that lesion angle had no impact on oneyear clinical outcomes, inconsistent with a past report in the bare metal stent era [7]. This might be derived from the better mechanical performance of second-generation DES with thinner struts and improved cell design. Some reports have demonstrated that injuries of larger size and ISA would not have healed well, resulting in incomplete healing of vessel injuries and persistent strut malapposition [8]. Therefore, we need further studies to evaluate the long-term clinical outcomes and clinical significance of optical coherence tomography (OCT) findings in patients with angled lesions. 


\section{Conclusions}

ISR is not uncommon and several risk factors have been reported as predisposing factors for such unwanted outcomes. Stenting of an angled lesion is associated with a higher incidence of ISR as compared to a nonangled lesion. It has been reported previously in the major coronary arteries. To our knowledge, our case is the first to report such an effect on an anomalous left main coronary artery.

\section{Additional Information \\ Disclosures}

Human subjects: Consent was obtained by all participants in this study. Conflicts of interest: In compliance with the ICMJE uniform disclosure form, all authors declare the following: Payment/services info: All authors have declared that no financial support was received from any organization for the submitted work. Financial relationships: All authors have declared that they have no financial relationships at present or within the previous three years with any organizations that might have an interest in the submitted work. Other relationships: All authors have declared that there are no other relationships or activities that could appear to have influenced the submitted work.

\section{References}

1. Buccheri D, Piraino D, Andolina G, Cortese B: Understanding and managing in-stent restenosis: a review of clinical data, from pathogenesis to treatment. J Thorac Dis. 2016, 8:1150. 10.21037/jtd.2016.10.93

2. Ino $\mathrm{Y}$, Kubo $\mathrm{T}$, Kitabata $\mathrm{H}$, et al.: Impact of hinge motion on in-stent restenosis after sirolimus-eluting stent implantation [EPub]. Circ J. 2011, 75:1878-1884. 10.1253/circj.cj-10-1182

3. Colombo A, Orlic D, Stankovic G, et al.: Preliminary observations regarding angiographic pattern of restenosis after rapamycin-eluting stent implantation. Circulation. 2003, 6:2178-2180.

10.1161/01.CIR.0000070592.04766.36

4. Al Bugami S, Althobaiti MW, Alrahemi J, Alsaiedi AJ, Kashkari WA: Coronary intervention of an anomalous left main coronary artery arising from the right sinus of Valsalva presented as acute coronary syndrome. JCCR. 2016, 5:1-3. 10.15406/jccr.2016.05.00184

5. Her AY, Shin ES: Current management of in-stent restenosis . Korean Circ J. 2018, 48:337-349. 10.4070/kcj.2018.0103

6. Pleva L, Kukla P, Hlinomaz O: Treatment of coronary in-stent restenosis: a systematic review . J Geriatr Cardiol. 2018, 15:173-184. 10.11909/j.issn.1671-5411.2018.02.007

7. Gomez-Lara J, Heo JH, Brugaletta S, et al.: Risk of target lesion failure in relationship to vessel angiographic geometry and stent conformability using the second generation of drug-eluting stents. Am Heart J. 2011, 162:1062-1079. 10.1016/j.ahj.2011.09.010

8. Gutierrez-Chico JL, Wykrzykowska J, Nuesch E, et al.: Vascular tissue reaction to acute malapposition in human coronary arteries. Sequential assessment with optical coherence tomography. Circ Cardiovasc Interv. 2012, 5:20-29. 10.1161/CIRCINTERVENTIONS.111.965301 